\title{
Identification of Neuroglycan C and Interacting Partners as Potential Susceptibility Genes for Schizophrenia in a Southern Chinese Population
}

Hon-Cheong So, ${ }^{1}$ Pui Y. Fong, ${ }^{2}$ Ronald Y.L. Chen, ${ }^{1}$ Tomy C.K. Hui, ${ }^{1}$ Mandy Y.M. Ng, ${ }^{2}$ Stacey S. Cherny, ${ }^{1,2,4}$ William W.M. Mak, ${ }^{2,3,5}$ Eric F.C. Cheung, ${ }^{6}$ Raymond C.K. Chan, ${ }^{1,7,8}$ Eric Y.H. Chen, ${ }^{1}$ Tao $\mathrm{Li}^{9}{ }^{9}$ and Pak C. Sham ${ }^{1,2,4 *}$

\author{
${ }^{1}$ Department of Psychiatry, University of Hong Kong, Hong Kong SAR, China \\ ${ }^{2}$ Genome Research Centre, University of Hong Kong, Hong Kong SAR, China \\ ${ }^{3}$ Department of Biochemistry, University of Hong Kong, Hong Kong SAR, China \\ ${ }^{4}$ The State Key Laboratory of Brain and Cognitive Sciences, University of Hong Kong, Hong Kong SAR, China \\ ${ }^{5}$ Hong Kong Biotechnology Education Resource Centre, Hong Kong SAR, China \\ ${ }^{6}$ Castle Peak Hospital, Hong Kong SAR, China \\ ${ }^{7}$ Neuropsychology and Applied Cognitive Neuroscience Laboratory, Institute of Psychology, Chinese Academy of Sciences, Beijing, China \\ ${ }^{8}$ Key Laboratory of Mental Health, Institute of Psychology, Chinese Academy of Sciences, Beijing, China \\ ${ }^{9}$ Division of Psychological Medicine and SGDP Centre, Institute of Psychiatry, King's College, London, UK
}

Received 3 July 2008; Accepted 2 March 2009

Chromosome 3p was reported by previous studies as one of the regions showing strong evidence of linkage with schizophrenia. We performed a fine-mapping association study of a 6-Mb high$\mathrm{LD}$ and gene-rich region on $3 p$ in a Southern Chinese sample of 489 schizophrenia patients and 519 controls to search for susceptibility genes. In the initial screen, 4 SNPs out of the 144 tag SNPs genotyped were nominally significant $(P<0.05)$. One of the most significant SNPs (rs3732530, $P=0.0048$ ) was a non-synonymous SNP in the neuroglycan C (NGC, also known as CSPG5) gene, which belongs to the neuregulin family. The gene prioritization program Endeavor ranked NGC 8th out of the 129 genes in the 6-Mb region and the highest among the genes within the same LD block. Further genotyping of NGC revealed 3 more SNPs to be nominally associated with schizophrenia. Three other genes (NRG1, ErbB3, ErbB4) involved in the neuregulin pathways were subsequently genotyped. Interaction analysis by multifactor dimensionality reduction (MDR) revealed a significant two-SNP interaction between NGC and NRG1 $(P=0.015)$ and three-SNP interactions between $N R G 1$ and $E r b B 4(P=0.009)$. The gene NGC is exclusively expressed in the brain. It is implicated in neurodevelopment in rats and was previously shown to promote neurite outgrowth. Methamphetamine, a drug that may induce psychotic symptoms, was reported to alter the expression of NGC. Taken together, these results suggest that NGC may be a novel candidate gene, and neuregulin signaling pathways may play an important role in schizophrenia. $\odot 2009$ Wiley-Liss, Inc.

Key words: neuregulin-1; CSPG5; ErbB4; genetic association; interaction

\section{How to Cite this Article:}

So H-C, Fong PY, Chen RYL, Hui TCK, Ng

MYM, Cherny SS, Mak WWM, Cheung EFC,

Chan RCK, Chen EYH, Li T, Sham PC. 2010.

Identification of Neuroglycan C and

Interacting Partners as Potential

Susceptibility Genes for Schizophrenia in a

Southern Chinese Population.

Am J Med Genet Part B 153B:103-113.

Additional Supporting Information may be found in the online version of this article.

Grant sponsor: University of Hong Kong Strategic Research Theme on Genomics; Grant sponsor: Hong Kong Research Grants Council General Research Fund; Grant number: 757905.

Hon-Cheong So and Pui Y. Fong contributed equally to this work.

${ }^{\star}$ Correspondence to:

Pak C. Sham, Department of Psychiatry, 10/F Laboratory Block, LKS Faculty of Medicine, University of Hong Kong, Pokfulam, Hong Kong SAR, China. E-mail: pcsham@hkucc.hku.hk

Published online 14 April 2009 in Wiley InterScience

(www.interscience.wiley.com)

DOI 10.1002/ajmg.b.30961 


\section{INTRODUCTION}

Schizophrenia (SZ [MIM 181500]) is a severe, common neurodevelopmental psychiatric disorder affecting nearly $1 \%$ of the human population [Sawa and Snyder, 2002]. Although the disorder has high heritability (around $80 \%$ as shown by twin studies) [Cardno and Gottesman, 2000], the overall evidence from family and linkage studies indicates complex inheritance with the involvement of multiple genetic loci. Linkage and association studies have so far identified a few susceptibility genes of modest effect, including neuregulin 1 (NRG1), dysbindin (DTNBP1), and D-amino-acid oxidase (DAAO) [Harrison and Weinberger, 2005].

Chromosome $3 \mathrm{p}$ is one region with evidence of linkage with SZ, first implicated by a genome-wide linkage scan [Pulver et al., 1995] which identified $3 \mathrm{p}$ and $8 \mathrm{p}$ to have the two highest LOD scores (2.34 and 3.00, respectively). Another linkage study on targeted chromosomal regions yielded a two-point LOD score of $\sim 1.5$ in support of linkage to 3p [Maziade et al., 2001]. The best evidence of linkage to 3 p came from the meta-analysis performed by Lewis et al. [2003]. This meta-analysis included data from 20 schizophrenia genome scans and employed a rank-based approach to combine evidence from different scans. The second bin on chromosome $3 p$ showed strong statistical significance for linkage, ranking 2nd in the unweighted analysis and 3rd in the weighted analysis (in which each study was weighted by the square root of its sample size).

In this study, we attempt to screen chromosome $3 p$ for susceptibility genes for SZ in a Southern Chinese sample of 489 schizophrenia patients and 519 controls. We decided to concentrate on the 47-53 Mb region of 3p, which has high levels of linkage disequilibrium (LD) and gene density (see Fig. 1). The region was interrogated by tag SNPs selected using a clustering algorithm [Ao et al., 2005] which has been further improved by using both $\mathrm{LD}$ and biological information in the selection process [Sham et al., 2007]. Using these tools, we have conducted a highdensity association study of this $47-53 \mathrm{Mb}$ region of $3 p$ to search for SZ susceptibility genes. Candidate genes that contain significant SNPs from this screen were then subjected to further exploration which included the genotyping of additional SNPs in these genes and their interacting partners.

\section{MATERIALS AND METHODS}

\section{Case-Control Sample}

Four hundred eighty-nine patients with schizophrenia diagnosed by DSM-IV were recruited from mental hospitals in Hong Kong. Five hundred nineteen controls without serious mental disorders were recruited from the community. Details of sample collection can be found elsewhere [Chen et al., 2001].

\section{Assay Design and Genotyping}

We employed the Sequenom MassARRAYTM system for assay design and genotyping, using SpectroDESIGNER software version 2.0.0.17. Genotyping was carried out by primer extension and MALDI-TOF mass spectrometry. For quality control, 20 samples were duplicated and 4 negative controls were included in each 384well plate. A successful assay was defined as one with (i) $\geq 90 \%$ of genotyping calls; and (ii) control genotypes not significantly deviating from the Hardy-Weinberg equilibrium $(P>0.01)$.

\section{Initial Association Screen}

For tag SNP selection, we downloaded genotype data on 1693 SNPs in the $3 p$ region typed on the HapMap Asian population (JPT and CHB), data release 2005-03_16a_phaseI (Build 34). The CLUSTAG [Ao et al., 2005] program, which employs an agglomerative clustering algorithm, was used for the selection of tag SNPs, with the $\mathrm{R}^{2}$ threshold set at 0.8 . If a SNP failed in the assay design, it was replaced by the second best SNP within the same cluster. From the 814 common SNPs (those of minor allele frequency $>5 \%$ ), we selected 218 tag SNPs and successfully designed genotyping assays for 214 of them. Of these, 162 SNPs genotyped passed the quality control criteria. The average genotype call rate was $96 \%$. Eighteen

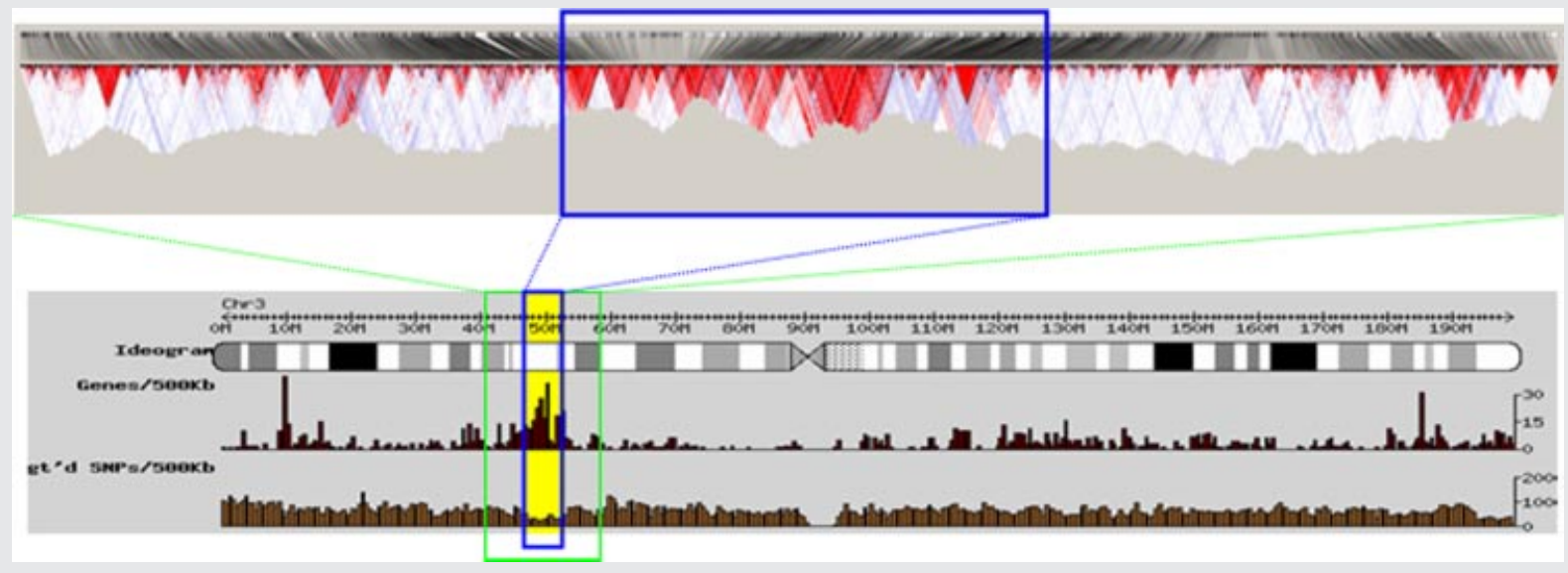

FIG. 1. The top figure is a LD plot of 41-59 Mb on chromosome 3. The plot shows a high LD region in the middle [ $47-53 \mathrm{Mb}$, boxed region]. The figure below is the ideogram of chromosome 3 with plots of gene density and SNP density. The 6-Mb high LD region is highlighted and shows a high gene density. [Color figure can be viewed in the online issue, which is available at www.interscience.wiley.com.] 
SNPs with minor allele frequency under $1 \%$ were discarded, leaving 144 markers for the association analyses. To ensure that the remaining markers still captured the variation in the $3 p$ region adequately, we reassessed the coverage of these 144 tag SNPs using Haploview. The proportion of the genetic variation captured was calculated with reference to HapMap release 21. We employed aggressive tagging with 2- and 3-marker haplotype tags. The original 144 tag SNPs captured $76 \%$ of the alleles in the linkage region at $r$-squared $\geq 0.8$. The average $R^{2}$ is 0.948 .

\section{Statistical Analyses for Association}

The programs WHAP (ver 2.06) [Saxena et al., 2007] (http:// pngu.mgh.harvard.edu/ purcell/whap/) and UNPHASED (ver 3.09) [Dudbridge, 2003] were used to screen all the 144 markers for both allelic and haplotype-based association. Genotype data were phased and haplotype frequencies were estimated using a standard E-M approach implemented in both programs. The programs performed a regression-based haplotype association test for all haplotypes. Moving window analyses were performed with single marker and with two to four consecutive markers as haplotypes. The false discovery rate (FDR) approach [Benjamini and Hochberg, 1995] was used to correct for multiple testing. Odds ratios and confidence intervals were calculated for the significant markers. LD statistics between markers were calculated and represented graphically by HaploView [Barrett et al., 2005].

\section{Prioritization of Genes Using Bioinformatics Tools}

To incorporate biological significance into the consideration of potential candidate genes, we used the software Endeavor [Aerts et al., 2006] to rank all the genes included in our initial screen. Endeavor prioritizes candidate genes underlying diseases or biological pathways by their similarity to a set of "training genes" which are specified by the user. Training genes are those with previous evidence for association with a particular disease or pathway. The program assumes that genes involved in the same disease or pathway are likely to share features with the known candidates. The program employs up to 12 different data sources, such as pathway membership in KEGG, annotations in Gene Ontology, sequence similarity and protein interactions to assess the properties of the training set. The final ranking is obtained by combining all data sources using order statistics. Endeavor is among the most extensively validated software designed for this purpose. For example, it has been validated by leave-one-out cross-validations using 703 disease and pathway genes, and by in vivo studies in zebrafish [Aerts et al., 2006]. In the present study, a training set was selected from the SZgene database (http://www.schizophreniaforum.org/res/sczgene/default.asp accessed 17th September 2007) and a review article on SZ genetics by Straub and Weinberger [2006]. Genes in the "Top Results" list from SZgene, all of which have at least one variant showing a nominally significant result in meta-analyses, were selected, as well as those listed in Table I in Straub and Weinberger's review (list of training genes is shown in Table I). To ensure the reliability of the rankings, we also repeated our analyses leaving one of the training genes out each time. This ensures that the results would not be seriously affected by individual training genes and protects against the risk that some training genes may later turn out to have no association with schizophrenia. We included all genes in the 47-53 Mb region on chromosome $3 \mathrm{p}$ as the testing set.

\section{Weighted FDR Analysis}

The rankings obtained from Endeavor were also used to weight the $P$-values obtained from the initial screen of the linkage region in a weighted FDR approach [Roeder et al., 2006]. The weight is based on the rankings of the 60 genes containing the genotyped markers. Different degrees of emphasis on biological information could be specified using different functions of the rankings; we used weights that were either directly proportional to ranking (linear weights) or to ranking squared (quadratic weights). Variants belonging to the same gene were given the same weighting. SNPs lying outside a gene were regarded as belonging to the nearest gene, provided that the gene was within $50 \mathrm{~kb}$ of the SNP.

\section{Follow-Up Analyses of Identified Genes}

For follow-up analyses, we used the WCLUSTAG [Sham et al., 2007] program for selection of additional tag SNPs within the most promising gene from the initial screen (which was neuroglycan C, see below). WCLUSTAG allows users to adjust the clustering threshold according to the location or presumed functional significance of the SNPs. In this study, the $\mathrm{R}^{2}$ tagging threshold was set to be 1.0 for SNPs in coding regions, 0.8 for SNPs within $5^{\prime} \mathrm{UTR}$, $3^{\prime} \mathrm{UTR}, 5^{\prime}$ upstream and $3^{\prime}$ downstream regions, and 0.3 for introns. We also genotyped possible interacting partners of neuroglycan $\mathrm{C}$ (NGC), namely NRG1, ErbB3, and ErbB4. NRG1 is the first member in the neuregulin family and is one of the most established susceptibility genes for SZ [Harrison and Law, 2006]. ErbB3 is a direct interacting partner of $N G C$ while both $E r b B 3$ and $E r b B 4$ are binding receptors for NRG1 (see Discussion Section).

Potential interactions among genetic markers in the same or different genes were assessed by multifactor dimensionality reduction (MDR) [Ritchie et al., 2001; Hahn et al., 2003], a model-free approach for detecting interactions between loci in association studies. MDR reduces the multilocus genotype data to one dimension by classifying each multilocus genotype as either high-risk or low-risk by the ratio of affected individuals to unaffected individuals with that genotype. We used the standard 10-fold cross validation to evaluate the accuracy of prediction of 2-SNP and 3 -SNP combinations and permutation tests to calculate empirical $P$-values. In order to test whether the interaction is significant over the main effects and whether the results are similar under a parametric approach, we also carried out logistic regression for interacting SNP pairs identified by MDR. The SNPs were coded in 0 , 1 , and 2 under the assumption of an additive model. The overall significance of interaction was assessed by a likelihood ratio test, comparing the full model (with all main effects and interaction terms) with the reduced model (with only the main effects).

\section{Bioinformatics Analyses of Candidate Genes}

We looked for functional domains that could be relevant to the disease for SNPs that showed statistically significant association. 
TABLE I. Training Set of Genes for Endeavor Analysis (Alternative Names for Genes Are Shown in Brackets)

Gene name

Neuregulin-1

Disrupted in schizophrenia 1

Regulator of G-protein signaling 4

Proline oxidase, mitochondrial precursor (Proline dehydrogenase)

Dysbindin (Dystrobrevin-binding protein 1)

D-amino-acid oxidase

Catechol 0-methyltransferase

D(3) dopamine receptor

Neuronal acetylcholine receptor subunit alpha-? precursor

$\mathrm{RAC}$-alpha serine/threonine-protein kinase (protein kinase B)

$\mathrm{D}(2)$ dopamine receptor (dopamine D2 receptor)

Metabotropic glutamate receptor 3 precursor

Serine/threonine-protein phosphatase 2B catalytic subunit gamma

Glutamate decarboxylase 1

Receptor tyro sine-protein kinase erbB-4 precursor

Fasciculation and elongation protein zeta 1

Thioredoxin domain-containing protein 5 precursor

Orofacial cleft 1 candidate 1

D-amino acid oxidase activator (protein G72)

Glutamate [NMDA] receptor subunit epsilon 2 precursor ( $\mathrm{N}$-methyl D-aspartate receptor subtype 2B)

Gamma-aminobutyric-acid receptor subunit beta-2 precursor

Tryptophan 5-hydroxylase 1

Sodium-dependent serotonin transporter [5HT transporter]

Interleukin-1 beta precursor

Dopamine receptor D4

Methylenetetrahydrofolatereductase

Plexin-A2 precursor (Semaphorin receptor OCT)

Apolipoprotein E precursor

$\mathrm{D}(1 \mathrm{~A})$ dopamine receptor

Haptoglobin precursor

Cellular tumor antigen p53 (tumor suppressor p53)

Gene symbol

NRG1

DISC1

RGS4

PRODH

DTNBP1

$D A O$ (DAAO)

COMT

DRD3

CHRNA?

AKT1 (PKB)

DRD2

GRM3

PPP3CC

GAD1

ERBB4

FEZ1

TXNDC5 (MUTED)

OFCC1 (MRDS1)

$D A O A$ (G?2)

GRIN2B (NMDAR2B)

GABRB2

TPH1

SLC6A4

IL1B

DRD4

MTHFR

PLXNA2

$A P O E$

DRD1

$H P$

TP53 (P53)
SNP sequences were downloaded from public databases. The sequences were submitted to Pfam [Finn et al., 2006], a public protein family database. Pfam has a large collection of multiple sequence alignments and hidden Markov models covering many common protein domains and families. The Wise2 software package (http://www.ebi.ac.uk/Wise2/) was used to blast DNA and protein sequences. Multiple species alignment was obtained for the domains to which our significant markers belong. For the gene of interest, the domain structure of its protein was obtained from UniProt (Universal Protein Resource) (http:// www.pir.uniprot.org/). Gene expression information was obtained from the Stanford Microarray Database (http://genome-www5. stanford.edu/).

\section{RESULTS}

\section{Association Screen Identified NGC as a Promising Candidate Gene for Schizophrenia}

In single marker association analyses, four SNPs were found to be nominally significant at $P<0.05$ (Fig. 2 and Table II), although the global permutation $P$-value of the most significant of the 144 tests was only 0.3107 . The best two markers rs $3732530(P=0.0048)$ and rs4858867 $(P=0.0040)$ were situated in exon 2 in neuroglycan $C$ (NGC, also known as chondroitin sulfate proteoglycan 5 , or CSPG5) and intron 13 in microtubule associated protein 4 (MAP4), respectively. The $\mathrm{R}^{2}$ between these 2 markers is 0.246 . The false discovery rate (or $q$-value) for the top 2 markers was 0.343 . $P$-values for all 144 markers in the initial screen are shown in Supplementary Table I.

We further screened all possible haplotypes with sizes of two, three, and four markers (Supplementary Table II). Haplotype analyses did not reveal any results with stronger significance than single locus analysis, with the best results being for haplotypes that clustered around the most significant SNPs in single-marker analyses. The best haplotype was formed by the markers rs3732530 and rs4858867 $(P=0.0066)$.

We examined the LD structure of the associated regions based on HapMap data and noted that most of the significant signals from single-marker and haplotype analyses were clustered around an LD block containing the genes NGC, SMARCC1, DHX30, and MAP4. Our results suggested associations to 


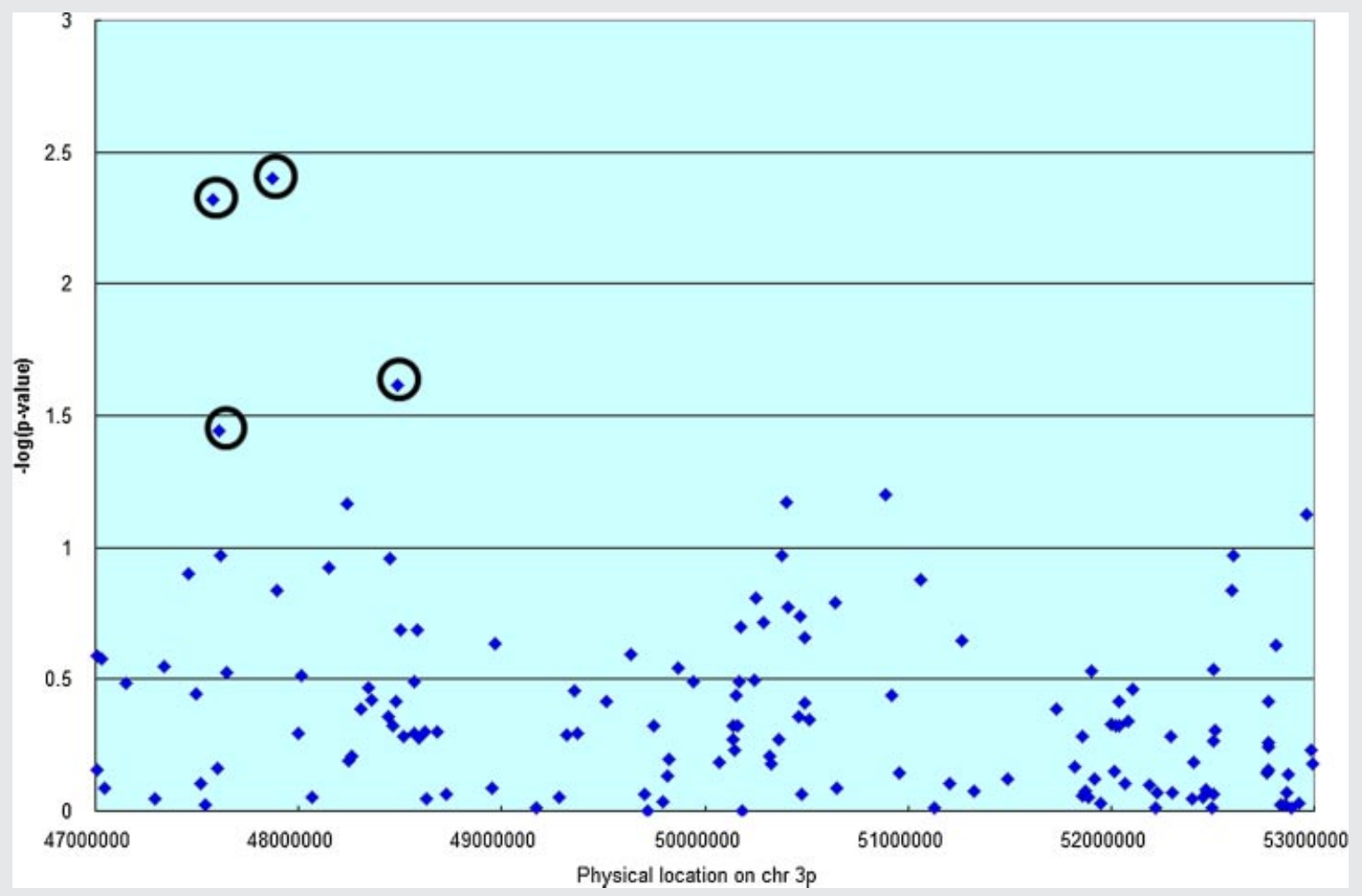

FIG. 2. Statistical significance of the 144 markers in the initial association screen. The four markers showing nominal significance $[P<0.05]$ are circled. [Color figure can be viewed in the online issue, which is available at www.interscience.wiley.com.]

this block, but could not distinguish the exact gene implicated in the disease due to high LD in the region. Hence we made use of the results from Endeavor to further prioritize genes in this region.

Using all 31 training genes, Endeavor ranked NGC 8th, MAP4 29th, DHX30 57th, and SMARCC1 67th out of the 129 genes in the $6 \mathrm{MB}$ region scanned (Table III). The NGC SNP rs3732530 had the lowest weighted $P$-value among the 144 genotyped SNPs. The weighted FDRs for rs3732530 were 0.411 and 0.302 under linear and quadratic weighting schemes, respectively. We repeated the analysis leaving each of the training genes out and calculated the average ranks. They were in general similar to the original ranks. The average rank of NGC was 9th and the actual leave-oneout rank ranged from 6 th to 15 th. The marker with the lowest weighted $P$-value using the average ranks was again rs3732530, having weighted FDRs of 0.413 and 0.303 respectively under linear and quadratic weighting schemes. We also calculated the weighted $P$-values in the least favorable scenario when NGC had the lowest leave-one-out rank (15th). Notably, the NGC SNP rs3732530 still achieved the lowest weighted $P$-value among the 144 genotyped markers.

As NGC was ranked by Endeavor as the most functionally relevant among the four genes in the implicated LD block and previous biological experiments also suggested a role of $N G C$ in neurodevelopment (see Discussion Section), we believed that it is the most promising candidate in the region and further genotyped this gene and its interacting partners.

\section{Follow-Up and MDR Analyses of NGC, NRG1,}

\section{ErbB3, and ErbB4}

In the follow-up phase, four out of seven markers tested within NGC showed nominally significant signals $(P<0.05)$. The best SNP in this gene ( $r$ 3732530) survived permutation correction for multiple testing within the gene (corrected $P=0.024$ ). Two haplotypes were also nominally significant and the best haplotype was formed by markers rs3732530 and rs12489865 $(P=0.0179)$ (Table IV, Supplementary Tables III and IV).

For ErbB4, two markers (rs2371276 and rs13032249) were found to be significant with $P$-values 0.0063 and 0.0465 , respectively. Four haplotypes also showed $P<0.05$. Although the best ErbB4 marker did not withstand permutation correction, the $q$-values for the best two markers (NGC_5 and ErbB4_11) in the second stage of association analysis (considering all 58 SNPs) were 0.183 . None of the NRG1 markers was significant when considered individually. However, three haplotypes showed nominal significance. The $P$-value for the most significant haplotype was 0.011 , although this was not significant after permutation correction. No significant result was found in the three markers genotyped in ErbB3 (Table IV).

In MDR analyses, five marker combinations were shown to be statistically significant by permutation tests which corrected for the inclusion of multiple SNPs. The results were significant at an FDR of 0.05. The best two-SNP model was composed of variants from NGC and NRG1, while the best three-SNP model included SNPs from 
TABLE II. Initial Association Screen of 144 Markers [Only Significant Results Shown]

\begin{tabular}{|c|c|c|c|c|c|c|c|}
\hline \multirow[b]{2}{*}{$\begin{array}{l}\text { Marker } \\
12\end{array}$} & & \multicolumn{2}{|c|}{ Allele } & & & \\
\hline & $\begin{array}{l}\text { Gene } \\
\text { NGC } \\
\text { rs3732530 }\end{array}$ & $\begin{array}{l}\text { Cases } \\
\text { Controls }\end{array}$ & $\begin{array}{c}\mathbf{A} \\
605(0.646) \\
572(0.584)\end{array}$ & $\begin{array}{c}\text { C } \\
331(0.354) \\
408(0.416)\end{array}$ & $\begin{array}{l}\text { LR chisq } \\
7.952\end{array}$ & $\begin{array}{l}\boldsymbol{P} \text {-value } \\
0.0048\end{array}$ & $\begin{array}{c}\text { OR }[\mathbf{9 5 \%} \mathrm{CI}) \\
1.30(1.08-1.57)\end{array}$ \\
\hline & & & & & & & \\
\hline $\begin{array}{l}\text { Marker } \\
14\end{array}$ & $\begin{array}{c}\text { Gene } \\
\text { SMARCC1 } \\
\text { rs } 4274776\end{array}$ & $\begin{array}{l}\text { Cases } \\
\text { Controls }\end{array}$ & $\begin{array}{c}\mathbf{C} \\
271(0.281) \\
329(0.324)\end{array}$ & $\begin{array}{c}\mathbf{T} \\
695(0.720) \\
687(0.676)\end{array}$ & $\begin{array}{c}\text { LR chisq } \\
4.400\end{array}$ & $\begin{array}{l}P \text {-value } \\
0.0359\end{array}$ & $\begin{array}{c}\mathbf{O R}(\mathbf{9 5 \%} \% \mathbf{C l}) \\
1.23(1.01-1.49)\end{array}$ \\
\hline & & & & & & & \\
\hline $\begin{array}{l}\text { Marker } \\
17\end{array}$ & $\begin{array}{l}\quad \text { Gene } \\
\text { MAP4 } \\
\text { rs485886? }\end{array}$ & $\begin{array}{l}\text { Cases } \\
\text { Controls }\end{array}$ & $\begin{array}{c}\text { C } \\
777(0.873) \\
762(0.825)\end{array}$ & $\begin{array}{c}\mathbf{T} \\
113(0.127) \\
162(0.175)\end{array}$ & $\begin{array}{c}\text { LR chisq } \\
8.287\end{array}$ & $\begin{array}{l}\boldsymbol{P} \text {-value } \\
0.0040\end{array}$ & $\begin{array}{c}\text { OR }(95 \% \mathrm{CI}) \\
1.46(1.13-1.90)\end{array}$ \\
\hline & & & & & & & \\
\hline $\begin{array}{l}\text { Marker } \\
33\end{array}$ & $\begin{array}{l}\text { Gene } \\
\text { SCOTIN } \\
\text { rs6442126 }\end{array}$ & $\begin{array}{l}\text { Cases } \\
\text { Controls }\end{array}$ & $\begin{array}{c}\mathbf{A} \\
795(0.848) \\
822(0.809)\end{array}$ & $\begin{array}{c}\mathbf{T} \\
143(0.153) \\
194(0.191)\end{array}$ & $\begin{array}{l}\text { LR chisq } \\
5.084\end{array}$ & $\begin{array}{l}\boldsymbol{P} \text {-value } \\
0.0241\end{array}$ & $\begin{array}{c}\text { OR }[\mathbf{9 5 \%} \% \mathbf{C I}) \\
1.31(1.04-1.66)\end{array}$ \\
\hline
\end{tabular}

LR chisq, likelihood ratio chi-square; NGC, neuroglycan C (also named CSPG5, chondroitin sulfate proteoglycan 5); SMARCC1, SWI/SNF related, matrix associated, actin dependent regulator of chromatin, subfamily c, member 1 ; MAP4, microtubule associated protein 4.

ErbB4 and NRG1. Only the best 2-SNP model comprising NGC and NRG1 SNPs was also significant when logistic regression was employed (Table V).

\section{Bioinformatics Analysis for NGC}

None of the significant NGCSNPs had flanking sequences that lie in any structural units, except one, rs3732530, which was found to be in the chondroitin sulfate attachment domain (accession number PF06566 in the Pfam database). This SNP is a non-synonymous $\mathrm{SNP}$, in which the change of allele from $\mathrm{T}$ to $\mathrm{G}$ results in an amino acid change from Val to Gly. The sequence of this domain is highly conserved among mouse, rat, human, and chicken, with an additional leucine- and proline-rich sequence attached to the chondroitin sulfate chains in chicken. This domain together with the cytoplasmic domain form the major part of the NGC protein.

\section{DISCUSSION}

Using a fine-mapping strategy and a range of statistical tools such as WCLUSTAG, weighted FDR and MDR, we identified neuroglycan $\mathrm{C}(N G C)$, a membrane-spanning chondroitin sulfate proteoglycan, which is exclusively expressed in the brain, as a novel candidate gene for SZ. This finding is supported by both statistical and biological evidence.

In the association analyses, a non-synonymous SNP in NGC (rs3732530) showed a moderately high level of significance which survived permutation correction considering all the examined variants in the gene. Although a global permutation test for all
144 markers in the first screening stage did not show significance, the weighted FDR for the best two markers (in NGC and MAP4) was only 0.223 , using quadratic weighting according to the rankings from Endeavor. It is noteworthy that NGC ranked 8th out of the 129 genes in the 6-MB region scanned and the highest among the genes lying in the same LD block.

Importantly, a large body of evidence from biological experiments also supports a role for NGC in the pathogenesis of SZ. NGC is exclusively expressed in the brain, and was discovered in the developing rat brain. NGC is first expressed at embryonic day 16 and the expression level continues to rise with a peak at the early postnatal period, coinciding with the period of active neurite formation and synaptogenesis in the rat brain. Expression of the gene decreases as the brain matures [Watanabe et al., 1995]. NGC is expressed in association with axons and dendrites in the CNS during development [Schumacher et al., 1997; Aono et al., 2000]. Nakanishi et al. [2006] showed that a recombinant ectodomain of NGC core protein enhances neurite outgrowth in rat neurons, supporting the idea that $N G C$ is involved in neurodevelopment.

In addition, $C A L E B$ (chicken acidic leucine-rich EGF-like domain containing brain protein), a chick homolog of NGC, demonstrates biological functions associated with pathogenesis of schizophrenia. Expression of CALEB is restricted to the developing and adult nervous system. The component containing chondrotin sulfate was expressed in the embryonic nervous system and downregulated in the adult. $C A L E B$ is involved in neurite formation; antibodies against $C A L E B$ result in impairment of neurite extension in a permissive environment [Schumacher et al., 1997]. Another 
TABLE III. Endeavor Ranks of All Genes in the 6 Mb Region Scanned

\begin{tabular}{|c|c|c|}
\hline Gene symbol & Overall rank & Average rank \\
\hline GRM2 & 1 & 1.42 \\
\hline PLXNB1 & 2 & 1.84 \\
\hline SLC26A6 & 3 & 4.16 \\
\hline MST1 & 4 & 4.06 \\
\hline ITIH4 & 5 & 5.65 \\
\hline MST1R & 6 & 5.74 \\
\hline GNAI2 & 7 & 6.39 \\
\hline$N G C$ & 8 & 8.90 \\
\hline$B S N$ & 9 & 9.13 \\
\hline IMPDH2 & 10 & 11.55 \\
\hline$A M T$ & 11 & 11.58 \\
\hline CAMKV & 12 & 16.23 \\
\hline SEMA3B & 13 & 14.45 \\
\hline$\angle A M B 2$ & 14 & 12.61 \\
\hline COLPA1 & 15 & 15.35 \\
\hline МАРКАРКЗ & 16 & 14.97 \\
\hline ITIH1 & 17 & 19.84 \\
\hline USP4 & 18 & 23.39 \\
\hline SETD2 & 19 & 20.90 \\
\hline STAB1 & 20 & 19.45 \\
\hline$D A G 1$ & 21 & 17.90 \\
\hline GNL3 & 22 & 21.97 \\
\hline TLR9 & 23 & 22.23 \\
\hline PRKAR2A & 24 & 22.84 \\
\hline SHISA5 & 25 & 26.45 \\
\hline CDC25A & 26 & 28.48 \\
\hline$B A P 1$ & 27 & 27.00 \\
\hline NBEAL2 & 28 & 30.77 \\
\hline MAP4 & 29 & 31.68 \\
\hline RASSF1 & 30 & 30.61 \\
\hline$A C Y 1$ & 31 & 32.16 \\
\hline QARS & 32 & 34.52 \\
\hline RHOA & 33 & 30.81 \\
\hline DUSP7 & 34 & 33.06 \\
\hline ZMYND10 & 35 & 37.48 \\
\hline HYAL1 & 36 & 38.97 \\
\hline DOCK3 & 37 & 38.00 \\
\hline SCAP & 38 & 38.00 \\
\hline TUSC4 & 39 & 46.42 \\
\hline QRICH1 & 40 & 40.35 \\
\hline HYAL2 & 41 & 41.16 \\
\hline CACNA2D2 & 42 & 44.52 \\
\hline $\mathrm{NISCH}$ & 43 & 46.55 \\
\hline$A R I H 2$ & 44 & 45.42 \\
\hline PCBP4 & 45 & 40.23 \\
\hline RAD54L2 & 46 & 49.19 \\
\hline UQCRC1 & 47 & 41.65 \\
\hline SLC25A20 & 48 & 50.48 \\
\hline TREX1 & 49 & 49.65 \\
\hline USP19 & 50 & 62.19 \\
\hline PBRM1 & 51 & 53.26 \\
\hline SEMA3F & 52 & 49.74 \\
\hline UBE1L & 53 & 56.26 \\
\hline P4HTM & 54 & 52.32 \\
\hline IHPK1 & 55 & 52.84 \\
\hline SLC38A3 & 56 & 54.71 \\
\hline DHX30 & 57 & 55.16 \\
\hline
\end{tabular}

TABLE III. [Continued]

\begin{tabular}{|c|c|c|}
\hline Gene symbol & Overall rank & Average rank \\
\hline TEX264 & 58 & 62.32 \\
\hline TUSC2 & 59 & 59.19 \\
\hline $\mathrm{CISH}$ & 60 & 60.77 \\
\hline HYAL3 & 61 & 56.13 \\
\hline$A B H D 14 A$ & 62 & 51.32 \\
\hline SFMBT1 & 64 & 65.48 \\
\hline HEMK1 & 65 & 67.32 \\
\hline SEMA3G & 66 & 64.03 \\
\hline SMARCC1 & 67 & 64.00 \\
\hline ITIH3 & 68 & 69.68 \\
\hline GPX1 & 69 & 59.00 \\
\hline RBM5 & 70 & 75.35 \\
\hline IHPK2 & 71 & 71.13 \\
\hline NEK4 & 72 & 78.29 \\
\hline KIFg & 73 & 78.00 \\
\hline PPM1M & 74 & 80.84 \\
\hline GLYCTK & 75 & 74.74 \\
\hline$K L H L 18$ & 76 & 75.35 \\
\hline TNNC1 & 77 & 72.35 \\
\hline MON1A & 78 & 75.68 \\
\hline GNAT1 & 79 & 76.90 \\
\hline PARP3 & 80 & 75.29 \\
\hline VPRBP & 81 & 82.26 \\
\hline PTPN23 & 82 & 78.19 \\
\hline NT5DC2 & 83 & 81.77 \\
\hline RPL29 & 84 & 83.00 \\
\hline TMEM89 & 85 & 90.32 \\
\hline RNF123 & 86 & 85.10 \\
\hline CAMP & 87 & 77.19 \\
\hline$A P E H$ & 88 & 88.77 \\
\hline TMEM115 & 89 & 86.68 \\
\hline WDR51A & 90 & 90.97 \\
\hline $\operatorname{cCDC} 36$ & 91 & 89.81 \\
\hline GLT8D1 & 92 & 86.35 \\
\hline$R B M 15 B$ & 93 & 93.19 \\
\hline RBM6 & 94 & 96.90 \\
\hline TRAIP & 95 & 96.26 \\
\hline AMIG03 & 96 & 96.16 \\
\hline C3orf>1 & 97 & 97.90 \\
\hline WDR6 & 98 & 100.35 \\
\hline$A B H D 14 B$ & 99 & 101.32 \\
\hline FBXW12 & 100 & 93.35 \\
\hline NICN1 & 101 & 101.84 \\
\hline TCTA & 102 & 104.10 \\
\hline PFKFB4 & 103 & 107.68 \\
\hline SPCS1 & 104 & 102.55 \\
\hline C3orf62 & 105 & 105.03 \\
\hline GPR62 & 106 & 93.61 \\
\hline PHFP & 107 & 107.00 \\
\hline ALAS1 & 108 & 108.23 \\
\hline NME6 & 109 & 106.35 \\
\hline$C C D C>1$ & 110 & 109.16 \\
\hline CYB561D2 & 111 & 112.87 \\
\hline RRPg & 112 & 118.06 \\
\hline ZNF589 & 113 & 115.00 \\
\hline GMPPB & 114 & 112.97 \\
\hline DALRD3 & 115 & 115.32 \\
\hline TMEM113 & 116 & 115.39 \\
\hline
\end{tabular}


TABLE III. (Continued)

$\begin{array}{lcc}\text { Gene symbol } & \text { Overall rank } & \text { Average rank } \\ \text { CCDC51 } & 117 & 112.19 \\ \text { C3orf54 } & 118 & 117.65 \\ \text { TMEM103 } & 119 & 118.74 \\ \text { IDCF2 } & 120 & 119.35 \\ \text { UCN2 } & 121 & 118.97 \\ \text { DNAH1 } & 122 & 119.45 \\ \text { KLHDC8B } & 123 & 123.35 \\ \text { IDCF1 } & 124 & 123.90 \\ \text { C3orf60 } & 125 & 124.61 \\ \text { C3orf45 } & 126 & 125.10 \\ \text { CDH29 } & 127 & 127.16 \\ \text { STGC3 } & 128 & 127.81 \\ \text { C3orf18 } & 129 & 125.23\end{array}$

study [Juttner et al., 2005] showed that CALEB-deficient mice exhibit impaired synapse function in early postnatal stages.

NGChas been implicated in the effects of methamphetamine and cocaine, two drugs that are known to produce psychotic symptoms such as paranoid delusions and hallucinations. Ishikawa et al. [2006] reported that repeated administration of methamphetamine significantly increased mRNA levels of $N G C$ in numerous rat brain areas, such as frontal cortex, nucleus accumbens and hippocampus. Moreover, NGC protein levels in the nucleus accumbens increased in rats repeatedly treated by methamphetamine. Up-regulation in both mRNA and protein levels of NGC was also found in chronic cocaine-treated rats [Toda et al., 2002].

$N G C$ possesses an EGF-like extracellular domain and is an active growth factor. It is considered to be the sixth member of the neuregulin family, as it acts as a direct ligand for ErbB3 tyrosine kinases that can transactivate ErbB2 [Kinugasa et al., 2004]. Neuregulin 1 (NRG1), the first member of the neuregulin family, is a strong candidate gene for SZ. It is involved in a large variety of processes that may be relevant to the pathogenesis of SZ, such as neuronal migration, oligodendrocyte development, myelin formation, and neurotransmitter receptor (particularly NMDA receptor) function [Corfas et al., 2004; Harrison and Law, 2006]. Being another member of the neuregulin family, $N G C$ might share some of the functional properties with NRG1 and hence may be involved in the pathogenesis of SZ.

NRG1 has been reported to be associated with SZ in different populations. The initial study on NRG1 by Stefansson et al. [2002] and many other studies [Yang et al., 2003; Corvin et al., 2004; Hall et al., 2004; Li et al., 2004, 2006; Tang et al., 2004; Zhao et al., 2004] identified SNPs and haplotypes near the $5^{\prime}$ end of the gene to be associated with SZ. On the other hand, the $3^{\prime}$ end region was also implicated in a few studies [Yang et al., 2003; Li et al., 2004; Petryshen et al., 2005], two involving Chinese samples [Yang

TABLE IV. All Markers Showing Nominal Significance $(P<0.05$ ) in Single SNP (Allelic or Genotypic) or Haplotype Analyses in the Follow-Up Phase

\begin{tabular}{|c|c|c|c|c|c|c|}
\hline \multirow[b]{2}{*}{ Marker number } & \multirow{3}{*}{ rs number } & & & & & \\
\hline & & Genotypic & Allelic & 2-marker & 3-marker & 4-marker \\
\hline \multicolumn{6}{|l|}{ NGC } & \\
\hline NGC 3 & rs10865948 & 0.090 & 0.029 & & & \\
\hline$N G C^{-} 4$ & rs11716779 & 0.230 & 0.538 & 0.040 & & \\
\hline$N G C 5$ & $r s 3732530$ & 0.007 & 0.005 & 0.018 & & \\
\hline$N G C^{-} 6$ & rs12489865 & 0.022 & 0.017 & & & \\
\hline$N G C^{-}>$ & rs3755637 & 0.091 & 0.046 & & & \\
\hline \multicolumn{7}{|l|}{ NRG1 } \\
\hline NRG1 5 & rs1531746 & 0.664 & 0.801 & 0.016 & & \\
\hline NRG1 6 & rs10503904 & 0.594 & 0.658 & & & \\
\hline NRG19 & rs6986716 & 0.243 & 0.094 & 0.011 & 0.020 & \\
\hline NRG1 10 & rs10503915 & 0.055 & 0.286 & & & \\
\hline NRG1_11 & rs7016691 & 0.928 & 0.711 & & & \\
\hline \multicolumn{7}{|l|}{ ErbB4 } \\
\hline ErbB4_10 & rs2272024 & 0.994 & 0.982 & 0.036 & & \\
\hline ErbB4 11 & rs2371276 & 0.002 & 0.006 & 0.033 & & 0.040 \\
\hline ErbB4 12 & rs10205553 & 0.834 & 0.945 & & & \\
\hline ErbB4 13 & rs6435659 & 0.432 & 0.986 & & & \\
\hline ErbB4_14 & rs6435660 & 0.067 & 0.132 & & & \\
\hline ErbB4 17 & rs6435665 & 0.241 & 0.118 & & & 0.017 \\
\hline ErbB4_18 & rs6435670 & 0.941 & 0.757 & & & \\
\hline ErbB4 19 & rs839530 & 0.551 & 0.278 & & & \\
\hline ErbB4 20 & rs12478950 & 0.227 & 0.265 & & & \\
\hline
\end{tabular}

$P$-values for haplotype analyses are shown next to the 1 st SNP in the sliding window.

$P$-values that are nominally significant $(<0.05)$ are in bold. For haplotype analyses, only nominally significant results are shown. 
TABLE V. Average Prediction Accuracies of the Best Combinations of SNPs Identified by MDR and Logistic Regression

SNP combination

2-SNP

$1 \mathrm{st}$

2nd

3rd

3-SNP

1st ErbB4_6, ErbB4 13, NRG18

2nd ErbB4 3, ErbB4 11, NRG1?

3rd ErbB4_6, ErbB4_13, NGC_6 rs number of SNPs

\author{
rs10865948, rs4733130 \\ rs2371276, rs839530 \\ rs2371276, rs?628631
}

rs10197225, rs6435659, rs2347504

rs3748962, rs2371276, rs1545961

rs10197225, rs6435659, rs12489865

\section{Testing accuracy}

0.5711

0.5642

0.5522

0.5754

0.5751

$0.561 ?$
MDR p

0.015

0.031

0.097

0.009

0.009

0.043
Logistic regression $P$

0.009

0.125

0.140

0.996

0.409

0.295

$P$-values for MDR were derived from 1,000 permutations.

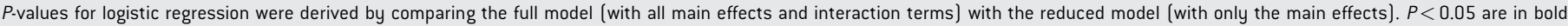

et al., 2003; Li et al., 2004]. The $5^{\prime}$ and $3^{\prime}$ regions containing susceptibility variants were designated regions $\mathrm{A}$ and $\mathrm{B}$ by Thomson et al. [2007]. Interestingly, the risk haplotypes identified in this study are between regions $\mathrm{A}$ and $\mathrm{B}$, a region of this large gene $(\sim 1.1 \mathrm{Mb})$ that most previous studies have not thoroughly screened, except Thomson et al. [2007] and Benzel et al. [2007]. Further comprehensive studies of NRG1 may be warranted, especially in non-Caucasian groups.

NRG1-ErbB signaling regulates a variety of processes that may be involved in SZ [Corfas et al., 2004]. ErbB3 and ErbB4 are hence good functional candidate genes for SZ. $E r b B 3$ is a direct interacting partner of NGC. Three microarray studies [Hakak et al., 2001; Tkachev et al., 2003; Aston et al., 2004] have shown that ErbB3, as a myelination-related gene, has altered expression in the post-mortem brains of schizophrenic patients. Oligodendrocyte dysfunction and disturbances in myelination are receiving increasing attention as important mechanisms underlying SZ [Karoutzou et al., 2008]. ErbB3 has also been examined previously in three association studies [Benzel et al., 2007; Kanazawa et al., 2007; Watanabe et al., 2007], but no susceptibility variants have been identified. We also failed to find any positive associations in ErbB3 in this study.

ErbB4 is another biological candidate for SZ and association studies have been performed on this gene. Silberberg et al. [2006] identified three SNPs in ErbB4 which showed marked differences in allele and genotype frequencies between schizophrenic patients and controls. Benzel et al. [2007] screened 109 SNPs in ErbB4 and found 14 SNPs with $P$-values $<0.05$ in either genotypic or allelic tests. Our study is the first to investigate ErbB4 polymorphisms in an Asian population. The two nominally significant SNPs and four-marker haplotype did not withstand permutation correction, but the FDR of the most significant ErbB4SNP was nevertheless 0.183, which can be considered suggestive, though not conclusive.

It should be noted that as the focus of the current study is on finemapping of chromosome $3 \mathrm{p}$ and discovery of NGC as a novel candidate gene, the genotyping coverage of the above interacting partners (NRG1, ErbB3, and ErbB4) may not be comprehensive enough. It is therefore possible that some susceptibility variants in these genes were not captured.

The finding in the MDR analyses of significant statistical interactions between SNPs at NGC and NRG1 is intriguing as these two genes are both members of the neuregulin family and share ErbB3 as a receptor. Thus it is plausible that they may play complementary or synergistic roles in the pathogenesis of schizophrenia, for example, in neurodevelopmental processes. Notably, the interaction between this pair of SNPs was also confirmed by logistic regression and the interaction was significant over the main effects. We also identified two sets of 3-locus interactions between NRG1 and ErbB4, consistent with the results of functional and association studies showing these two genes interact. Statistical epistasis between the Icelandic haplotype of NRG1 and a ErbB4 variant was previously reported by Norton et al. [2006]. Interestingly, we found statistical interaction between NGC and ErbB4 that was also modestly significant. Previous experiments showed that NGC directly binds to ErbB3 but not to ErbB4. Although NGC and ErbB4 may not be direct interacting partners, the two genes may be involved in complementary pathways related to brain development or other processes implicated in SZ. NGC, NRG1, and ErbB4 may be part of a network regulating a variety of processes involved in SZ.

Although these three-locus interactions were biologically plausible and supported by MDR, they did not show significance when logistic regression was used. This may be due to the different nature of the two approaches. MDR is non-parametric and completely model-free. MDR requires no mode of inheritance to be specified as any multilocus genotype can be formed as the high-risk or low-risk groups. It has high power to detect interactions in the absence of main effects [Ritchie et al., 2003]. Despite being more flexible, the genotype patterns identified by MDR may be very complex and difficult to interpret. On the other hand, logistic regression is a form of generalized linear model and does not involve partitioning of genotype combinations into high-risk and low-risk groups. Logistic regression may run into problems when dealing with large numbers of SNPs as the number of possible interaction terms increases rapidly with each additional SNP. Inclusion of too many independent variables with respect to the number of outcome events increases type 1 and type 2 errors [Peduzzi et al., 1996]. An inheritance model is often assumed to limit the number of terms in the regression model (we assumed an additive model in this study). However, logistic regression has an advantage over MDR in that it allows evaluation of interaction controlling for main effects. In view of the substantial differences between the two methods, some discrepancies between the test results are expected. Nevertheless, one should also alert to the possibility of false positives, particularly because we have included all SNPs regardless 
of whether they were independently associated with the disease. The interactions identified in this study should not be regarded as confirmatory and further association and functional studies are required to clarify the findings.

ErbB3 was not found in the interacting pairs in this study. However, its functional importance cannot be excluded. It is possible that the functional variant has not been captured (e.g., the variant may be a rare one) or there may be variants lying outside the gene which regulate its expression.

In summary, we have performed a high-density association screen of a high-LD, gene-rich region on chromosome $3 p$ and discovered NGC as a novel candidate gene for SZ. A role for NGC in $\mathrm{SZ}$ is supported by previous biological findings. Our methodology demonstrates the use of tag SNPs, bioinformatics tools, weighted FDR and MDR in association studies to identify new susceptibility genes for complex diseases. Although our study provides statistical evidence for the involvement of NGC and other candidate genes in SZ, our results cannot be considered conclusive. Further replications, preferably with larger samples and in other populations, are desirable for validation of our findings.

\section{ACKNOWLEDGMENTS}

The project was supported by a University of Hong Kong small project grant, the University of Hong Kong Strategic Research Theme on Genomics, and the Hong Kong Research Grants Council General Research Fund 757905. We are also grateful to C.L. Kwok, F. Lieh-Mak, J.H. Zhao, D. Collier, R. Murray, H.K. Cheung, P.C. $\mathrm{Lu}, \mathrm{C} . \mathrm{H}$. Yuen and C.K. Lin for their help in subject recruitment.

\section{REFERENCES}

Aerts S, Lambrechts D, Maity S, Van Loo P, Coessens B, De Smet F, Tranchevent LC, De Moor B, Marynen P, Hassan B, et al. 2006. Gene prioritization through genomic data fusion. Nat Biotechnol 24(5): 537-544.

Ao SI, Yip K, Ng M, Cheung D, Fong PY, Melhado I, Sham PC. 2005. CLUSTAG: Hierarchical clustering and graph methods for selecting tag SNPs. Bioinformatics 21(8):1735-1736.

Aono S, Keino H, Ono T, Yasuda Y, Tokita Y, Matsui F, Taniguchi M, Sonta S, Oohira A. 2000. Genomic organization and expression pattern of mouse neuroglycan $\mathrm{C}$ in the cerebellar development. J Biol Chem 275(1):337-342.

Aston C, Jiang L, Sokolov BP. 2004. Microarray analysis of postmortem temporal cortex from patients with schizophrenia. J Neurosci Res 77(6): 858-866.

Barrett JC, Fry B, Maller J, Daly MJ. 2005. Haploview: Analysis and visualization of LD and haplotype maps. Bioinformatics 21(2):263-265.

Benjamini Y, Hochberg Y. 1995. Controlling the false discovery rate: A practical and powerful approach to multiple testing. J R Stat Soc 57: 289-300.

Benzel I, Bansal A, Browning BL, Galwey NW, Maycox PR, McGinnis R, Smart D, St Clair D, Yates P, Purvis I. 2007. Interactions among genes in the ErbB-Neuregulin signalling network are associated with increased susceptibility to schizophrenia. Behav Brain Funct 3:31.

Cardno AG, Gottesman II. 2000. Twin studies of schizophrenia: From bowand-arrow concordances to star wars $\mathrm{Mx}$ and functional genomics. Am J Med Genet 97(1):12-17.
Chen RY, Sham P, Chen EY, Li T, Cheung EF, Hui TC, Kwok CL, Lieh-Mak F, Zhao JH, Collier D, et al. 2001. No association between T102C polymorphism of serotonin-2A receptor gene and clinical phenotypes of Chinese schizophrenic patients. Psychiatry Res 105(3): $175-185$.

Corfas G, Roy K, Buxbaum JD. 2004. Neuregulin 1-erbB signaling and the molecular/cellular basis of schizophrenia. Nat Neurosci 7(6):575-580.

Corvin AP, Morris DW, McGhee K, Schwaiger S, Scully P, Quinn J, Meagher D, Clair DS, Waddington JL, Gill M. 2004. Confirmation and refinement of an 'at-risk' haplotype for schizophrenia suggests the EST cluster, Hs.97362, as a potential susceptibility gene at the Neuregulin-1 locus. Mol Psychiatry 9(2):208-213.

Dudbridge F. 2003. Pedigree disequilibrium tests for multilocus haplotypes. Genet Epidemiol 25(2):115-121.

Finn RD, Mistry J, Schuster-Bockler B, Griffiths-Jones S, Hollich V, Lassmann T, Moxon S, Marshall M, Khanna A, Durbin R, et al. 2006. Pfam: Clans, web tools and services. Nucleic Acids Res 34(Database issue):D247-D251.

Hahn LW, Ritchie MD, Moore JH. 2003. Multifactor dimensionality reduction software for detecting gene-gene and gene-environment interactions. Bioinformatics 19(3):376-382.

Hakak Y, Walker JR, Li C, Wong WH, Davis KL, Buxbaum JD, Haroutunian V, Fienberg AA. 2001. Genome-wide expression analysis reveals dysregulation of myelination-related genes in chronic schizophrenia. Proc Natl Acad Sci USA 98(8):4746-4751.

Hall D, Gogos JA, Karayiorgou M. 2004. The contribution of three strong candidate schizophrenia susceptibility genes in demographically distinct populations. Genes Brain Behav 3(4):240-248.

Harrison PJ, Law AJ. 2006. Neuregulin 1 and schizophrenia: Genetics, gene expression, and neurobiology. Biol Psychiatry 60(2):132-140.

Harrison PJ, Weinberger DR. 2005. Schizophrenia genes, gene expression, and neuropathology: On the matter of their convergence. Mol Psychiatry 10(1):40-68, image 5.

Ishikawa K, Nitta A, Mizoguchi H, Mohri A, Murai R, Miyamoto Y, Noda Y, Kitaichi K, Yamada K, Nabeshima T. 2006. Effects of single and repeated administration of methamphetamine or morphine on neuroglycan $\mathrm{C}$ gene expression in the rat brain. Int J Neuropsychopharmacol 9(4):407-415.

Juttner R, More MI, Das D, Babich A, Meier J, Henning M, Erdmann B, Mu Ller EC, Otto A, Grantyn R, et al. 2005. Impaired synapse function during postnatal development in the absence of CALEB, an EGF-like protein processed by neuronal activity. Neuron 46(2):233-245.

Kanazawa T, Glatt SJ, Tsutsumi A, Kikuyama H, Koh J, Yoneda H, Tsuang MT. 2007. Schizophrenia is not associated with the functional candidate gene ERB B3: Results from a case-control study. Am J Med Genet Part B 144B(1):113-116.

Karoutzou G, Emrich HM, Dietrich DE. 2008. The myelin-pathogenesis puzzle in schizophrenia: A literature review. Mol Psychiatry 13:245-260.

Kinugasa Y, Ishiguro H, Tokita Y, Oohira A, Ohmoto H, Higashiyama S. 2004. Neuroglycan C, a novel member of the neuregulin family. Biochem Biophys Res Commun 321(4):1045-1049.

Lewis CM, Levinson DF, Wise LH, DeLisi LE, Straub RE, Hovatta I, Williams NM, Schwab SG, Pulver AE, Faraone SV, et al. 2003. Genome scan meta-analysis of schizophrenia and bipolar disorder, part II: Schizophrenia. Am J Hum Genet 73(1):34-48.

Li T, Stefansson H, Gudfinnsson E, Cai G, Liu X, Murray RM, Steinthorsdottir V, Januel D, Gudnadottir VG, Petursson H, et al. 2004. Identification of a novel neuregulin 1 at-risk haplotype in Han schizophrenia Chinese patients, but no association with the Icelandic/Scottish risk haplotype. Mol Psychiatry 9(7):698-704. 
Li D, Collier DA, He L. 2006. Meta-analysis shows strong positive association of the neuregulin 1 (NRG1) gene with schizophrenia. Hum Mol Genet 15(12):1995-2002.

Maziade M, Roy MA, Rouillard E, Bissonnette L, Fournier JP, Roy A, Garneau Y, Montgrain N, Potvin A, Cliche D, et al. 2001. A search for specific and common susceptibility loci for schizophrenia and bipolar disorder: A linkage study in 13 target chromosomes. Mol Psychiatry 6(6):684-693.

Nakanishi K, Aono S, Hirano K, Kuroda Y, Ida M, Tokita Y, Matsui F, Oohira A. 2006. Identification of neurite outgrowth-promoting domains of neuroglycan C, a brain-specific chondroitin sulfate proteoglycan, and involvement of phosphatidylinositol 3-kinase and protein kinase C signaling pathways in neuritogenesis. J Biol Chem 281(34):24970-24978.

Norton N, Moskvina V, Morris DW, Bray NJ, Zammit S, Williams NM, Williams HJ, Preece AC, Dwyer S, Wilkinson JC, et al. 2006. Evidence that interaction between neuregulin 1 and its receptor erbB4 increases susceptibility to schizophrenia. Am J Med Genet Part B 141B(1):96-101.

Peduzzi P, Concato J, Kemper E, Holford TR, Feinstein AR. 1996. A simulation study of the number of events per variable in logistic regression analysis. J Clin Epidemiol 49(12):1373-1379.

Petryshen TL, Middleton FA, Kirby A, Aldinger KA, Purcell S, Tahl AR, Morley CP, McGann L, Gentile KL, Rockwell GN, et al. 2005. Support for involvement of neuregulin 1 in schizophrenia pathophysiology. Mol Psychiatry 10(4):366-374, 328.

Pulver AE, Lasseter VK, Kasch L, Wolyniec P, Nestadt G, Blouin JL, Kimberland M, Babb R, Vourlis S, Chen H, et al. 1995. Schizophrenia: A genome scan targets chromosomes $3 p$ and $8 p$ as potential sites of susceptibility genes. Am J Med Genet 60(3):252-260.

Ritchie MD, Hahn LW, Roodi N, Bailey LR, Dupont WD, Parl FF, Moore JH. 2001. Multifactor-dimensionality reduction reveals high-order interactions among estrogen-metabolism genes in sporadic breast cancer. Am J Hum Genet 69(1):138-147.

Ritchie MD, Hahn LW, Moore JH. 2003. Power of multifactor dimensionality reduction for detecting gene-gene interactions in the presence of genotyping error, missing data, phenocopy, and genetic heterogeneity. Genet Epidemiol 24(2):150-157.

Roeder K, Bacanu SA, Wasserman L, Devlin B. 2006. Using linkage genome scans to improve power of association in genome scans. Am J Hum Genet 78(2):243-252.

Sawa A, Snyder SH. 2002. Schizophrenia: Diverse approaches to a complex disease. Science 296(5568):692-695.

Saxena R, Voight BF, Lyssenko V, Burtt NP, de Bakker PI, Chen H, Roix JJ, Kathiresan S, Hirschhorn JN, Daly MJ, et al. 2007. Genome-wide association analysis identifies loci for type 2 diabetes and triglyceride levels. Science 316(5829):1331-1336.
Schumacher S, Volkmer H, Buck F, Otto A, Tarnok A, Roth S, Rathjen FG. 1997. Chicken acidic leucine-rich EGF-like domain containing brain protein (CALEB), a neural member of the EGF family of differentiation factors, is implicated in neurite formation. J Cell Biol 136(4):895-906.

Sham PC, Ao SI, Kwan JS, Kao P, Cheung F, Fong PY, Ng MK. 2007. Combining functional and linkage disequilibrium information in the selection of tag SNPs. Bioinformatics 23(1):129-131.

Silberberg G, Darvasi A, Pinkas-Kramarski R, Navon R. 2006. The involvement of ErbB4 with schizophrenia: Association and expression studies. Am J Med Genet Part B 141B(2):142-148.

Stefansson H, Sigurdsson E, Steinthorsdottir V, Bjornsdottir S, Sigmundsson T, Ghosh S, Brynjolfsson J, Gunnarsdottir S, Ivarsson O, Chou TT, et al. 2002. Neuregulin 1 and susceptibility to schizophrenia. Am J Hum Genet 71(4):877-892.

Straub RE, Weinberger DR. 2006. Schizophrenia genes-Famine to feast. Biol Psychiatry 60(2):81-83.

Tang JX, Chen WY, He G, Zhou J, Gu NF, Feng GY, He L. 2004. Polymorphisms within 5' end of the Neuregulin 1 gene are genetically associated with schizophrenia in the Chinese population. Mol Psychiatry 9(1):11-12.

Thomson PA, Christoforou A, Morris SW, Adie E, Pickard BS, Porteous DJ, Muir WJ, Blackwood DH, Evans KL. 2007. Association of Neuregulin 1 with schizophrenia and bipolar disorder in a second cohort from the Scottish population. Mol Psychiatry 12(1):94-104.

Tkachev D, Mimmack ML, Ryan MM, Wayland M, Freeman T, Jones PB, Starkey M, Webster MJ, Yolken RH, Bahn S. 2003. Oligodendrocyte dysfunction in schizophrenia and bipolar disorder. Lancet 362(9386): 798-805.

Toda S, McGinty JF, Kalivas PW. 2002. Repeated cocaine administration alters the expression of genes in corticolimbic circuitry after a 3-week withdrawal: A DNA macroarray study. J Neurochem 82(5):1290-1299.

Watanabe E, Maeda N, Matsui F, Kushima Y, Noda M, Oohira A. 1995. Neuroglycan C, a novel membrane-spanning chondroitin sulfate proteoglycan that is restricted to the brain. J Biol Chem 270(45): 26876-26882.

Watanabe Y, Fukui N, Nunokawa A, Muratake T, Kaneko N, Kitamura H, Someya T. 2007. No association between the ERBB3 gene and schizophrenia in a Japanese population. Neurosci Res 57(4):574-578.

Yang JZ, Si TM, Ruan Y, Ling YS, Han YH, Wang XL, Zhou M, Zhang HY, Kong QM, Liu C, et al. 2003. Association study of neuregulin 1 gene with schizophrenia. Mol Psychiatry 8(7):706-709.

Zhao X, Shi Y, Tang J, Tang R, Yu L, Gu N, Feng G, Zhu S, Liu H, Xing Y, et al. 2004. A case control and family based association study of the neuregulin1 gene and schizophrenia. J Med Genet 41(1):31-34. 